of exercising anticipation of its carer's remote movements. Anticipation was signalled by the dog sitting at a window from which the outside world could be observed. The dog sat in the window on 55 per cent of the occasions when the return journey was under way, but only 5 per cent of the time when the carer (called Pam) was absent. For the videotaped observations, the timing of Pam's return was determined by a third party (using a cellular telephone) at random times: this, Sheldrake says, is when the dog's behaviour most clearly showed the knack of knowing when Pam had decided to return but had not yet embarked on the journey home.

By conceding that the data gathered during these observations are statistically significant, one does not sign up for Sheldrake's interpretation that the underlying mechanism is dog-Homo telepathy. Too many variables are uncontrolled. Did the accuracy of anticipation vary with the length of time elapsed since Pam's departure (suggesting that the dog used its sense of the passage of time to signal its sense of when return was due)? Were there people in the room with the dog (allowing them to communicate somehow with the eager waiter)? And while Jaytee appears to have been chosen for videotaping as a result of his acumen in earlier trials, does not the interpretation of his behaviour require an understanding of the variability of dogs' capacity for anticipation in general? The appendix in which these details are meticulously described is not so much a log of research under way as a record of one of those sets of observations preliminary to the design of properly controlled observations.

The remainder of the main text of this book, meanwhile, is curiously boring. It consists mostly of accounts, running to a few pages at the most, of how horses have been able to find their way home with an injured rider on their back, how cats have cried at the remote death of a human with whom they have been familiar, how dogs have howled when members of the family with which they live have been killed in action in some distant war, and even how people have been taken sick in sympathy with distant injured pets. Especially because people's fondness for their pets often takes the form of projecting onto them human or even superhuman perceptiveness, even more than 1,000 records on the Sheldrake website do not prove telepathy.

I doubt that Sheldrake will take the point. He makes plain his distaste for what he calls orthodox science, which is "all too often equated with a narrow-minded dogmatism that seeks to deny or debunk whatever does not fit in with the mechanistic view of the world". He is habitually courteous and cheerful, but holists of his ilk would not dream of letting controls get in the way of revealed truth.

John Maddox is at 9 Pitt Street, London W8 4NX, UK.

\section{Eccentricities of an everyday substance}

\section{$\mathrm{H}_{2} \mathrm{O}$ : A Biography of Water}

by Philip Ball

Weidenfeld \& Nicolson: 1999. 387 pp. $\mathfrak{E} 17.99$

\section{Frank H. Stillinger}

Linking human perceptions of water in mythology, cosmology, politics, literature and the physical and biological sciences (and pseudosciences) may seem to be an idiosyncratic objective. Yet that is just what Philip Ball, until recently a senior editor at Nature, has undertaken. With the possible exception of gold, there can be no other substance for which one could write such an engaging account of the profound historical influence exerted on this wide range of subjects. The author's panoramic knowledge, conveyed through a clear and often delightful writing style, makes attractive reading for a technically literate, but not necessarily expert, audience.

The narrative begins with the Big Bang and the subsequent expansion of the Universe that, in due course, produced the elements and chemical compounds, and supplied the early Earth with a chemical and physical ambience conducive to the spontaneous appearance of life. Ball traces our terrestrial geological chronology, and summarizes our present understanding of the role of continental, atmospheric and oceanic water transport in present-day meteorology. And in keeping with the burgeoning interest in planetary exploration in our Solar System, he summarizes the intriguing extraterrestrial evidence for water in both solid and liquid forms that might conceivably have spawned alternative life forms beyond Earth.

Although water has often been seen as 'unique' among liquids, labelling it as such is not especially informative, except in the most trivial sense. Nevertheless, it exhibits an impressive array of anomalies in its physical properties that might qualify it as 'eccentric'. Among these anomalies are the well-known expansion when it freezes at ordinary pressures, and the presence of a liquid-phase density maximum at $4^{\circ} \mathrm{C}$.

The author undertakes to explain, or at least to rationalize, these attributes in terms of the known structure of the water molecule, its resulting electrical asymmetry, and its propensity to engage in tetrahedral arrangements of hydrogen bonds with its own kind as neighbours. The last of these has been amusingly anthropomorphized with line drawings that should appeal to all ages: each water molecule has been rendered as a round-bodied elf whose two arms are destined to grab nearby elfin ankles. This device

^๑ 1999 Macmillan Magazines Ltd is used to introduce the reader to the structure of ordinary hexagonal ice and the forms displayed by snowflakes, and to the structures of high-pressure forms of ice.

In answering the question 'What is liquid water?' by looking at history, Ball has reflected the emergence and maturation of science itself. Beginning with the ancient Greek perception of the substance as one of the primal elements, the shifting answer has reflected specifically the rise of modern structural chemistry. But even in what one might call the 'modern' period (the past half-century, say), considerable revision has occurred to the details of how the liquid is geometrically organized by hydrogen bonding. Imaginative, but now clearly naive, pictures insisted that water should be viewed as modified

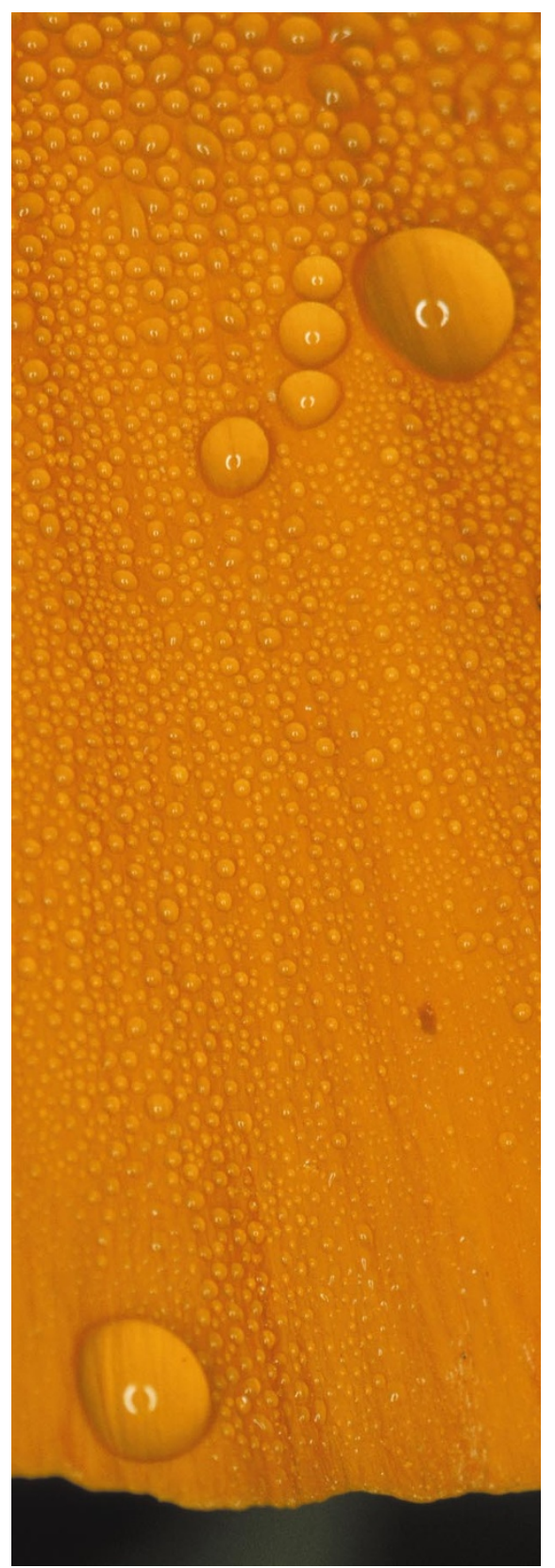

Dewdrops: a macroscopic network of molecules linked by frequent but transient hydrogen bonds. 
versions of ice (the 'iceberg' and 'interstitial ice' models), as a 'self clathrate', or as a distorted four-fold coordinated network of hydrogen bonds.

The present consensus seems to be that liquid water is a macroscopic network of molecules connected by frequent but transient hydrogen bonds, which allow unbonded neighbours to occur in numbers that vary with temperature and pressure. Attempts to identify unambiguous patterns of local molecular order that represent portions of the known ice polymorphs have generally proved to be unproductive. In any case, several independent computer simulations for liquid water, using only molecular equations of motion and estimates of the fundamental intermolecular interactions, confirm the random, defective-network viewpoint while automatically producing the characteristic thermodynamic water anomalies.

Quantitative modelling variations on this network viewpoint underlie recent studies directed at an intriguing possibility: does the regime of supercooled liquid water harbour a hidden first-order phase transition between two metastable liquids with different densities (to which the term 'polyamorphism' has recently been attached), along with an associated second critical point?

Water that is internal to living organisms differs from its pure bulk form. Aqueous biological fluids are electrolytes, and typically contain a wide array of biopolymers, nutrients and metabolites. As Ball emphasizes, intracellular water is forced to occupy a very crowded neighbourhood indeed, with available channels between the biopolymers being measured in nanometres, if not in ångströms. Consequently, most biological water is surface, or interfacial, water. Yet this aqueous solvent medium has vital roles in controlling the native folding patterns of proteins, and acting as a lubricant for the entire dynamic apparatus of life.

Quantitative details of this solvation role are still a bit hazy, but some broad themes have emerged from research. In particular, Ball provides an account of the present understanding of the 'hydrophobic interaction' phenomenon, which contributes significantly to protein folding and to the structural stability of membranes by driving together hydrocarbon-like molecular moieties.

It is in connection with Ball's discussion of the interfacial properties of liquid water that I would raise a minor quibble with what otherwise appears to be an accurate text. This concerns the comparison of the range of hydrophobic surface effects to the diameter of human hair. By using random samples from my own head as a basis, I estimate that this statement appears to be out by about two orders of magnitude.

As an object of research scrutiny, water has arguably attracted more than its fair share of questionable, or even absurd, claims. But like it or not, these scraps of pathological science constitute a legitimate part of the biography of water, so Ball provides detailed accounts of three notorious examples: the 'polywater' episode, which began in the former Soviet Union in the 1960s; 'cold fusion', announced by Stanley Pons and Martin Fleischmann in the United States in March 1989; and the alleged homoeopathic phenomenon reported a year earlier by Jacques Benveniste and collaborators for repeatedly diluted solutions of anti-IgE antibodies. Perhaps the inclusion of the last of these was inevitable, given that Nature itself was an active but nervous participant in its dissemination.

Some might judge that these and other less prominent water aberrations detract from the scientific legitimacy of the field, but the author intimates correctly that the situation deserves a more positive spin. First, the responses to these challenges to technical common sense affirm the health and vigour of the scientific method. Second, occasional bizarre claims can be interpreted as far-out indicators that creative imagination is widely at play, and this, when suitably filtered, provides the driving force for progress.

I read this book while Hurricane Floyd was inflicting its epic watery damage on the Atlantic coastline of the United States. This was a forceful reminder that understanding, let alone predicting, phenomena of all scales in our water-rich environment is still woefully inadequate. The mind wanders into musing about what a sequel to this book written at the end of the next century might reveal that this volume cannot. But for now, Ball's contribution is a delightful status report.

Frank H. Stillinger is at Bell Laboratories, Lucent Technologies, 600 Mountain Avenue, Murray Hill, New Jersey 07974, USA.

\section{New rhythms of our lives}

\section{The 24 Hour Society}

by Leon Kreitzman

Profile: 1999.176 pp. 116.99

\section{Paolo Sassone-Corsi}

The moment that Neil Armstrong and Buzz Aldrin took humankind's first steps on the Moon, 30 years ago, characterizes one of the peaks of human technological progress. The event concerned everyone from an intellectual and moral point of view, but the technological impact on our everyday lives appeared minimal. In those days we had no fax, e-mail, Internet or mobile phones. Although we had the capacity to send men walking on the Moon, we were still very slow

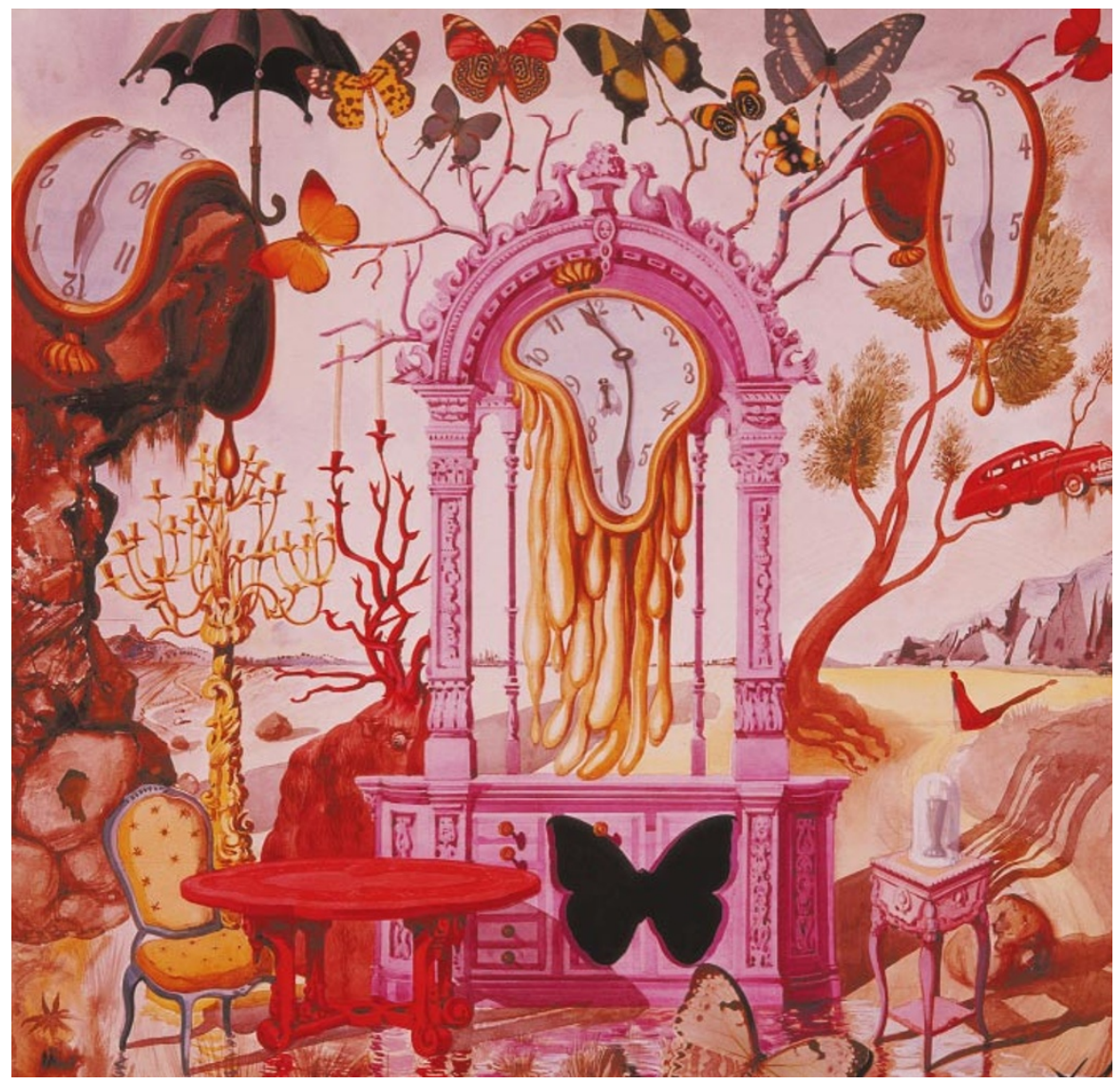

Dali on time: we take a more flexible view of the clock than did previous generations. 